\title{
Comparative studies on extracts from Hericium erinaceus by different polarity reagents to gain higher antioxidant activities
}

\author{
SHENGJUAN JIANG, YULIANG WANG and XIAOLONG ZHANG \\ College of Life Science, Anhui Science and Technology University, Fengyang, Anhui 233100, P.R. China
}

Received January 25, 2015; Accepted March 8, 2016

DOI: $10.3892 /$ etm.2016.3279

\begin{abstract}
Hericium erinaceus (H. erinaceus) is a source of exogenous antioxidants that has been traditionally used in China for the prevention and treatment of oxidative stress-associated disease. In the present study, the bioactive compounds of $H$. erinaceus were extracted with the following eight representative reagents: n-Hexane, xylene, chloroform, anhydrous ether, ethyl acetate, acetone, anhydrous ethanol and distilled water. The in vitro antioxidant activities were also evaluated. All of the extracted compounds exhibited reducing power and scavenging activity against 1-diphenyl-2-picrylhydrazyl (DPPH) and superoxide anion free radicals. In addition, the antioxidant capacities varied with the used chemical reagents and exhibited dose-dependent effects. Extracts from anhydrous ethanol, chloroform and acetone were capable of inhibiting lipid peroxidation. The anhydrous ethanol extracts were observed to have significant levels of antioxidant compounds since they had a strong reducing power, high scavenging rates against DPPH and superoxide anion-free radicals $(>90 \%)$, and high inhibition rates on lipid peroxidation $(>60 \%)$. The present study will provide reference data for the antioxidant applications of $H$. erinaceus in pharmaceutical use and disease prevention.
\end{abstract}

\section{Introduction}

Hericium erinaceus (H. erinaceus), a wood-rotting fungus, is a wild edible delicacy in China. Due to its edibility and functionality, the mushroom has attracted considerable attention. $H$. erinaceus is able to alleviate complex neurodegeneration, decrease gastric mucosal damage and significantly reduce the levels of plasma triglycerides (1). In addition, $H$. erinaceus possesses antioxidant activities, immunoregulatory activities and anti-cancer roles (1). H. erinaceus contains proteins,

Correspondence to: Dr Shengjuan Jiang, College of Life Science, Anhui Science and Technology University, 9 Donghua Road, Fengyang, Anhui 233100, P.R. China

E-mail: jiangsjahstu@126.com

Key words: Hericium erinaceus, antioxidant activity, extractions, polarity, homeostasis unsaturated fatty acids, carbohydrates and a variety of trace elements, including phosphorus, sulfur, calcium, magnesium, zinc, iron and copper (1). All the nutrients serve different important roles in the multiple physiological systems of the organism, including the nervous, digestive, circulatory and immune systems (1).

The physiological systems in the body should be balanced to maintain whole-body homeostasis. Once the balance is disturbed, a disease can occur (2). There is increasing evidence to indicate that oxidative stress is one of the causes of complicated diseases, including cancer and Alzheimer's disease in humans (3). For example, oxidative stress can contribute to tissue and cell injury and accelerate the proliferation of colorectal cancer cells (4). Reactive oxygen species (ROS) and lipid peroxides are damaging compounds involved in the oxidative stress process. An imbalance between ROS levels and antioxidant defenses occurs in various different diseases, such as Crohn's disease (5). Intake of antioxidants is important to sustain an adequate level in order to balance the ROS in vivo. A number of studies have demonstrated the close association between the prevention of ROS-associated diseases and intake of food rich in antioxidants, including mushrooms (6).

As a medicinal mushroom, $H$. erinaceus has abundant active substances that perform antioxidative roles. The ethanol and hot water extracts of $H$. erinaceus have antioxidant activities, including reducing ability, chelating effects on ferrous ions, 1-diphenyl-2-picrylhydrazyl (DPPH) free radical scavenging activity and inhibition of lipid peroxidation in emulsified corn oil or in emulsified egg yolk buffer $(6,7)$. Polysaccharides (water extracts) have a strong antioxidant activity in vitro, and they have been demonstrated to significantly increase the activity of antioxidant enzymes in vivo in a dose-dependent manner $(8,9)$. Therefore, $H$. erinaceus is a plentiful source of exogenous antioxidants, which may be considered important remedies for ameliorating pathological alterations in oxidative stress-associated disease. There is great diversity of active molecules in the fruit-body with different biological activities (10). The active ingredients in $H$. erinaceus can not be $100 \%$ extracted by a single reagent. Furthermore, different bioactive molecules have different solubilities in various solvents (11). Therefore, it is essential to evaluate the antioxidant activities of extracts produced by multiple representative solvents with different polarities.

The majority of the reports on antioxidant properties of $H$. erinaceus utilize ethanol, methanol and hot water 
for preparation of extracts, individually. However, there are various extraction conditions available in the literature. Therefore, it is rather difficult to compare the activities of different extracts when using different extraction conditions. Additionally, there are various other representative solvents, which are usually used to extract bioactive compounds. Nevertheless, few previous studies have investigated them. Hence, the objective of the present study was to analyze the in vitro antioxidant properties of $H$. erinaceus extracts produced by eight reagents, including n-hexane, xylene, chloroform, anhydrous ether, ethyl acetate, acetone, anhydrous ethanol and distilled water.

\section{Materials and methods}

Material preparation. Fresh $H$. erinaceus mushrooms (Houza 19 strain) were obtained from the Institute of Edible Fungus at Anhui Science and Technology University (Fengyang, China) at a mature stage. The mushrooms were sorted, cleaned, washed in cold sterilized water and drained. The fresh mushrooms were then lyophilized and powdered with a mixer (Zhejiang Ronghao Industry and Trade Co., Ltd., Zhejiang, China). The extractions and analyses were immediately conducted following lyophilization. All chemicals and reagents used in the present study were purchased from Sinopharm Chemical Reagent Co., Ltd. (Shanghai, China) unless otherwise stated.

Preparation of crude extracts. The powder was then subjected to extraction by the following eight solvents: n-Hexane, xylene, chloroform, anhydrous ether, ethyl acetate, acetone, anhydrous ethanol and distilled water. The optimized extraction conditions were used as follows: $20 \mathrm{~g}$ powder and $100 \mathrm{ml}$ reagent solution $(100 \%)$ added into a flask and extracted at room temperature for $24 \mathrm{~h}$. This process was repeated twice more. The liquid was collected by centrifugation $(2,500 \mathrm{x} \mathrm{g}, 5 \mathrm{~min}$, room temperature), and then condensed by rotary evaporation at $50^{\circ} \mathrm{C}$. The extracts were put into an evaporating dish and dried in a water bath at $50^{\circ} \mathrm{C}$. The crude extracts were dissolved in methanol, yielding a series of sample solutions with different concentrations.

Determination of reducing power. The reducing power was determined by the method of Jiang et al (12). The $\mathrm{K}_{4} \mathrm{Fe}(\mathrm{CN})_{6}$ was generated after the antioxidants reacted with $\mathrm{K}_{3} \mathrm{Fe}(\mathrm{CN})_{6}$. Then $\mathrm{K}_{4} \mathrm{Fe}(\mathrm{CN})_{6}$ reacted with $\mathrm{FeCl}_{3}$ to produce Perl's Prussian blue $\left(\mathrm{Fe}_{4}\left[\mathrm{Fe}(\mathrm{CN})_{6}\right]_{3}\right)$ which had the maximum absorbance at $700 \mathrm{~nm}$. The reaction mixture containing $2.5 \mathrm{ml}$ different concentrations of samples in phosphate-buffered saline (0.2 M, pH 6.6) was incubated with $2.5 \mathrm{ml} \mathrm{K}_{3} \mathrm{Fe}(\mathrm{CN})_{6}(1 \%$, $\mathrm{w} / \mathrm{v}$ ) at $50^{\circ} \mathrm{C}$ for $20 \mathrm{~min}$. The reaction was terminated by adding $2.5 \mathrm{ml}$ trichloroacetic acid $(10 \%)$ and the mixture was centrifuged at $1,400 \mathrm{x}$ g for $10 \mathrm{~min}$. The supernatant $(2.5 \mathrm{ml})$ was mixed gently with $2.5 \mathrm{ml}$ distilled water and $0.5 \mathrm{ml} \mathrm{FeCl}_{3}(0.1 \%, \mathrm{w} / \mathrm{v})$ and the absorbance was measured at $700 \mathrm{~nm}$ against a blank sample (V-1200 spectrophotometer; Shanghai Meipuda Instrument Co., Ltd., Shanghai, China). A higher absorbance indicated a higher reducing power. The experiments were performed in triplicate and the data was the average of all three.
DPPH radical scavenging activity. The DPPH radical scavenging activity of the samples was measured according to the procedure described by Negro et al (13). The solution of DPPH (0.2 mM; Sigma-Aldrich, St. Louis, MO, USA) was dissolved in methanol and was freshly prepared prior to the measurements. In brief, the extracted solutions $(2 \mathrm{ml})$ were thoroughly mixed with $2 \mathrm{ml}$ DPPH solution and allowed to stand for $30 \mathrm{~min}$ in the dark. The blank control solution contained an equal volume of distilled water instead of the sample solution. The absorbance was measured at $517 \mathrm{~nm}$ (V-1200 spectrophotometer). Lower absorbance of the reaction mixture indicated higher free radical scavenging activity. The experiments were performed in triplicate and the data were averaged. The scavenging rate was calculated according to the following equation: DPPH scavenging rate $(\%)=\left(\mathrm{A}_{\mathrm{c}}-\mathrm{A}_{\mathrm{s}}\right) \times 100 / \mathrm{A}_{\mathrm{c}}$, in which $\mathrm{A}_{\mathrm{c}}$ was the absorbance of the control, and $\mathrm{A}_{\mathrm{s}}$ was the absorbance of the sample.

Superoxide anion radical scavenging activity. The superoxide radical scavenging activity of the samples was evaluated according to the method reported by Chen et al (14) with a slight modification. The extract produced by different solvents was dissolved in $2 \mathrm{ml}$ methanol to form varying concentrations. The $2 \mathrm{ml}$ extracts and $2 \mathrm{ml}$ Tris- $\mathrm{HCl}$ buffer $(50 \mathrm{mM}$, $\mathrm{pH}$ 8.2) were blended and incubated at $37^{\circ} \mathrm{C}$ for $20 \mathrm{~min}$. Next, the solution was mixed with $1 \mathrm{ml} 50 \mathrm{mM}$ pyrogallol solution (preheated to $37^{\circ} \mathrm{C}$ ) and the absorbance variation was detected within $2 \mathrm{~min}$ at $325 \mathrm{~nm}$ (UV762 ultraviolet and visible spectrophotometer; Shanghai Jingke Scientific Instrument Co., Ltd., Shanghai, China). For the blank control, the sample was replaced by Tris- $\mathrm{HCl}$ buffer. The scavenging rate was calculated within $2 \mathrm{~min}$ by the following equation: Scavenging rate $(\%)=\left(\Delta \mathrm{A}_{\mathrm{c}}-\Delta \mathrm{A}_{\mathrm{s}}\right) \times 100 / \Delta \mathrm{A}_{\mathrm{c}}$, in which $\Delta \mathrm{A}_{\mathrm{s}}$ and $\Delta \mathrm{A}_{\mathrm{c}}$ were the absorbance variations of the extractions and the control.

Inhibition of lipid peroxidation. The peroxide value (POV) was used to assess the inhibition ability of extracts against lipid peroxidation. POV was measured as described in the Standard Methods for the Analysis of Fats and Oils established by China (GB/T5538-2005) (15). Autoxidation caused the formation of peroxides, which in turn resulted in the release of iodine. The free iodine was then titrated with a sodium thiosulfate solution (15). To artificially induce a quick oxidation of the corn oil, all samples were marked and stored in an oven at $60^{\circ} \mathrm{C}$ for $120 \mathrm{~h}$. During this process of oxidation, the POVs of samples were determined every $24 \mathrm{~h}$ using the Standard Methods for the Analysis of Fats and Oils established by China (GB/T5538-2005) (15). In brief, $2 \mathrm{~g}$ oil sample (the minimal effective concentration of extracts was $5 \%$ ) was mixed with $50 \mathrm{ml}$ isooctane and acetic acid mixture $(2: 3, \mathrm{v} / \mathrm{v})$. Next, $0.5 \mathrm{ml}$ saturated potassium iodide solution was added to the reaction system. The mixture was left in the darkness for $3 \mathrm{~min}$ and the supernatant was titrated with sodium thiosulfate solution $(2 \mathrm{mM})$. The POV was expressed as milliequivalents of active oxygen per kilogram of oil (meq/kg). The experiment was performed in triplicate. The POV was calculated as follows: POV $(\mathrm{meq} / \mathrm{kg})=1,000 \times \Delta \mathrm{V} \times \mathrm{c} / \mathrm{w}$, in which the $\mathrm{c}$ was the molar concentration of sodium thiosulfate solution, and 
W was the mass of the oil sample. $\Delta \mathrm{V}$ was the volume variation of the sodium thiosulfate solution that was consumed by the sample and the reagent blank control.

Statistical analysis. The antioxidant data were analyzed using analysis of variance in the SPSS statistics software version 18.0 (SPSS, Inc., Chicago, IL, USA) for significance. The data are expressed as the mean \pm standard deviation. $\mathrm{P}<0.05$ and $\mathrm{P}<0.01$ were considered to indicate a statistical significant difference. All the graphs were prepared using Microsoft Excel 2003 (Microsoft, Redmond, WA, USA).

\section{Results}

Reducing power. The antioxidants donate electrons and convert the oxidized form of iron $\left(\mathrm{Fe}^{3+}\right)$ to the ferrous form $\left(\mathrm{Fe}^{2+}\right)$, which can be monitored by measuring the formation of Perl's Prussian blue at $700 \mathrm{~nm}$ (16). The efficacy of certain antioxidants is associated with their reducing power (17). In the present assay, the results demonstrated that the absorbance, which reflects the reducing power, increased with the concentration of extractions (Fig. 1). The reducing powers at a maximum concentration of $2 \mathrm{mg} / \mathrm{ml}$ ranged between 0.254 for $n$-Hexane and 0.982 for distilled water. The reducing powers of the extractions that were produced by distilled water and anhydrous ethanol yielded the higehst absorbance levels. Thus, it may be deduced that the aqueous and alcoholic extracts possess significant levels of antioxidant compounds. The reducing power of the compounds after n-hexane extraction was the lowest.

DPPH radical scavenging activity. The stable DPPH free radical has an absorption of $517 \mathrm{~nm}$ in methanol (18). It can be widely used to evaluate the free radical scavenging ability of natural compounds with the hydrogen-donating ability (16). The antioxidant donates protons to the DPPH radical and the absorption decreases with the discoloration of the initial purple compound. The radical scavenging activity increases as the absorption at $517 \mathrm{~nm}$ is decreased (19). The results demonstrated that the DPPH radical scavenging activities of all types of extractions were concentration-dependent (Fig. 2). The extractions at high doses exhibited significant scavenging effects on DPPH free radicals. The scavenging activity of different extracts at $2 \mathrm{mg} / \mathrm{ml}$ ranged from 28.2 to $93.4 \%$. The distilled water and anhydrous ethanol presented the highest DPPH scavenging activities, whereas, the anhydrous ether extracts had the lowest DPPH scavenging activities, whereas, the extractions obtained from the four solvents with the higher polarity had a stronger scavenging rate than those from the four solvents with the lower polarity. The effective components in H.erinaceus with different polarities could be extracted by the solvents with the corresponding polarities. Furthermore, the biological activities of the effective components were different when the polarities were different. The results indicated that the scavenging activity of the extractions was associated with the polarities of the used reagents, and, the stronger the polarity of the solvent was, the higher the DPPH scavenging activity of its extraction.

Superoxide anion radical scavenging activity. Superoxide anions are the most common free radicals in vivo generated in a variety of biological systems. Higher levels of superoxide

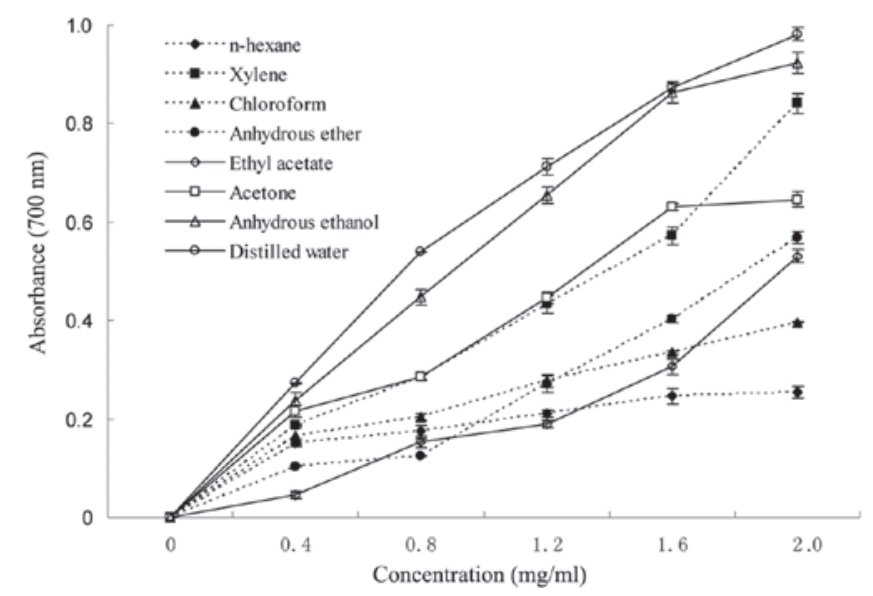

Figure 1. Reducing power of extracts from $H$. erinaceus produced by different reagents as a function of concentration. Data are presented as the mean \pm standard deviation $(n=3)$.

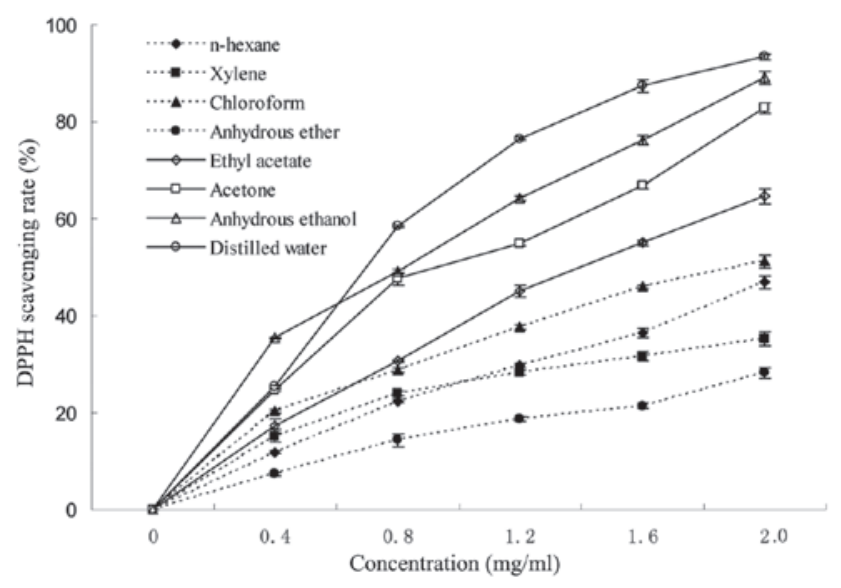

Figure 2. DPPH scavenging activity of extracts from H. erinaceus produced by different reagents as a function of concentration. Data are presented as the mean \pm standard deviation $(n=3)$. DPPH, 1-diphenyl-2-picrylhydrazyl.

anion radicals are regarded as the beginning of ROS accumulation in cells. In addition, they have been implicated in a number of human diseases, including cancer and diabetes (20). In the present study, the superoxide anion radical scavenging effects of different extractions exhibited a dose-dependent association (Fig. 3). The scavenging rate of different extractions at $10 \mathrm{mg} / \mathrm{ml}$ was in the range of $13.4-97.9 \%$. Furthermore, the extractions from anhydrous ethanol, n-hexane and chloroform had strong scavenging activities against superoxide anion radicals, whilst that of anhydrous ether was the weakest.

Lipid peroxidation. Edible oils can be autoxidized in the air. The peroxidation of lipids is a free radical driven chain reaction and various toxic secondary products are generated (21). In addition, these products are known to damage DNA and cell and organelle membranes, or cause cancers, such as liver cancer. Antioxidants terminate the lipid peroxidation chain reactions by removing free radical intermediates or inhibiting other oxidation reactions (22). Amongst the eight extractions, the anhydrous ethanol, chloroform and acetone extracts were capable of inhibiting lipid peroxidation in the corn oil system, as their POVs 
were lower than that of corn oil autoxidation (Fig. 4). The extractions did not significantly reduce the peroxide formation at the early stages (days 1-3) of the experiment. On the fourth day, the inhibition rates of anhydrous ethanol, chloroform and acetone extracts were $67.5,50$ and $10.2 \%$, respectively, as compared with the POV of corn oil auto-oxidation. On the fifth day, anhydrous ethanol, chloroform and acetone extracts caused 62, 71.2 and $54.1 \%$ reductions in the formation of peroxides, respectively compared with oil autooxidation. Following statistical analysis, the three extractions on days 4 and 5 were observed to significantly inhibit lipid peroxidation at a 0.01 significance level.

\section{Discussion}

H. erinaceus is a notable mushroom with medicinal values; it contains various bioactive compounds with drug efficacy. The nutrients in H. erinaceus include herinase (23) and isohericenone (24). In the present study, eight reagents with nonpolar, weak, middle and strong polarity were used to extract the active compounds from $H$. erinaceus.

The compounds in H. erinaceus have their own chemical structures and properties. They also have different solubilities in different chemical solvents (10). Thus, each extraction inevitably was a mixture of numerous constituents. Amongst the mixture, there were antioxidants and synergists, which increase the antioxidant roles. The extracts from $H$. erinaceus produced by eight reagents all exhibited a reducing power and scavenging activity against DPPH and superoxide anion free radicals. The antioxidant activities varied with the extraction concentrations and the chemical reagents that were used. The mixtures extracted by the solvents with similar polarity presented different specific activities. For example, the scavenging activities against DPPH and superoxide anion radical of anhydrous ether extraction were low, although it had a middle-level reducing power. The xylene extracts had a relatively high reducing power and superoxide anion radical scavenging activity, however, they did not effectively scavenge the DPPH radical. Similar results were observed for other reagents with nonpolar, weak and middle polarity. The results indicate that each extraction may have its own specific function in different antioxidant assays, since the extractions have complicated constituents that possess diverse chemical characteristics. In the reducing power and free radical scavenging assays, the activities of anhydrous ethanol and distilled water extractions were among the highest. This may indicate that polar ingredients contribute greatly to the antioxidant activities. However, the water extraction did not inhibit lipid peroxidation. It is possible that the efficient compounds were not fully extracted at the low extraction temperature that was used. Based on the results of the current study, anhydrous ethanol appears to be the most efficient extraction solvent that can be used to extract the effective antioxidant compounds in the food and pharmaceutical industries.

In the present study, four of the most frequently used methods were performed to detect the antioxidant activity of the extracts. Each method has a different mechanism, such as termination of the free-radical mediated chain reaction, hydrogen donation and elimination of peroxides (25). The results demonstrate that there is no universal method to give an accurate and comprehensive picture of an antioxidant profile (26). Thus, multiple assays based on different antioxidant mechanisms are crucial in order

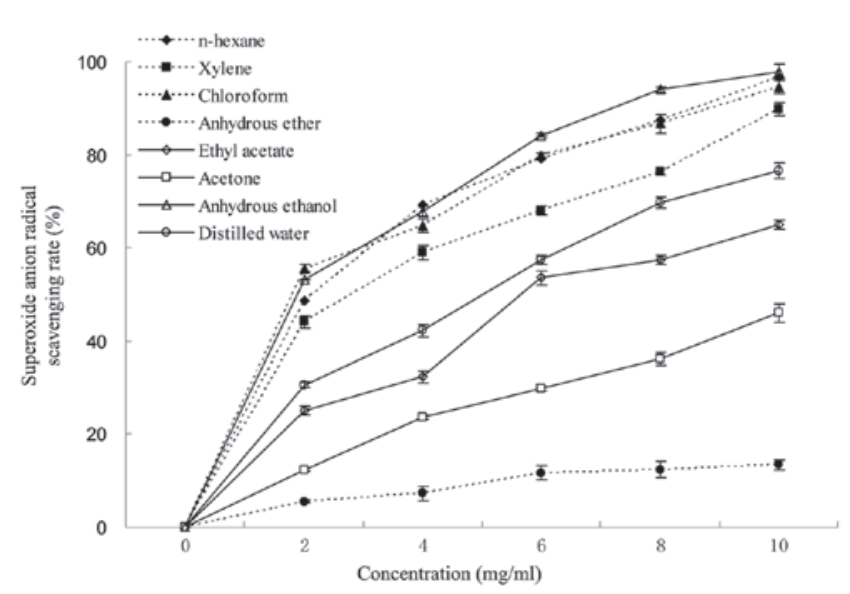

Figure 3. Superoxide anion radical scavenging activity of extracts from $H$. erinaceus produced by different reagents as a function of concentration. Data are presented as the mean \pm standard deviation $(n=3)$.

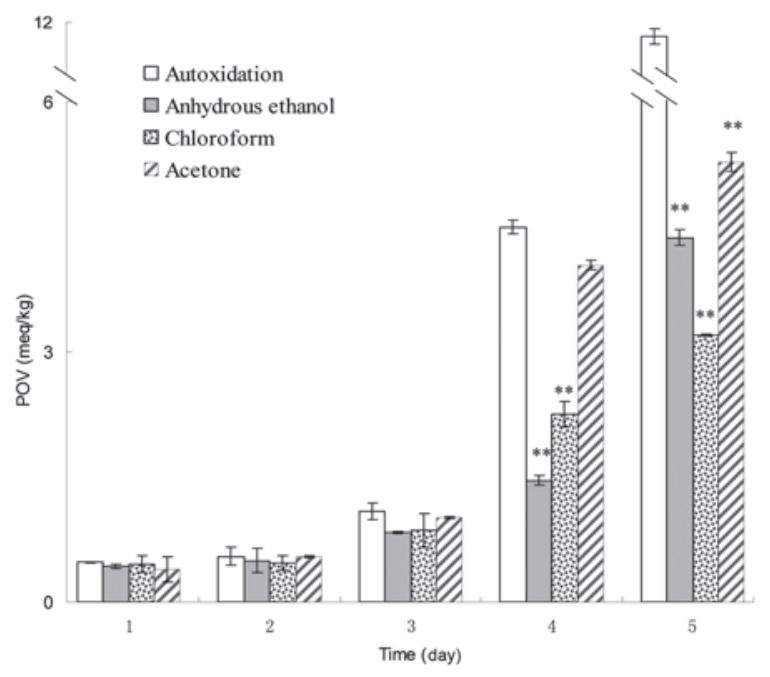

Figure 4. Peroxide value of extracts from $H$. erinaceus produced by different reagents as a function of concentration. Data are presented as the mean \pm standard deviation $(n=3) .{ }^{* *} \mathrm{P}<0.01$ vs. autoxidation group. POV, peroxide value.

to provide a reliable approach of measuring the antioxidant capacity of the extractions.

Under pathological conditions, free radicals and lipid peroxides damage the proteins, membranes and nucleic acids through numerous biological processes, thus giving rise to a variety of diseases, such as gastrointestinal mucosal diseases (27). Therefore, the demand for discovering novel compounds with good antioxidant activity is understandable (28). However, nowadays many people are in the sub-health status, which is an immediate stage between health and disease (29); and although the disease is in the early stages in these patients, the oxidative pressure in the body gradually increases and the antioxidant capacity gradually decreases (30). There is growing evidence that oxidative stress has a pivotal role in the pathogenesis of numerous diseases (31). The risk of diseases, including type 2 diabetes, atherosclerosis and cancer, is increasing. Therefore, the prevention of disease with potential natural, anti-oxidative compounds would be of great significance to human health. In 
the present study, extractions from $H$. erinaceus by different solvents have been demonstrated to have dose-dependent antioxidant activities. Since $H$. erinaceus is a source of natural bioactive compounds, its antioxidant compounds may be prospective protective agents that will help reduce the problem of suboptimal health statuses in humans and even reduce oxidative damage-associated diseases. The present study provides reference data for the application of $H$. erinaceus in the pharmaceutical industry and for disease prevention. Additionally, the broad medicinal values of mushrooms such as $H$. erinaceus have a promising future in alternative medicine. To understand the mechanisms behind the properties of the active ingredients, further study is required. Furthermore, a greater understanding of the structures and functions of the components that are responsible for the observed antioxidant activities is also required.

\section{Acknowledgements}

The present work was supported by the Foundation of Anhui Educational Commission of China (grant no. KJ2013A075), the Revitalization Plan of Anhui Province (grant no. gxyqZD2016222), and the National Natural Science Foundation of China (grant no. 31250002).

\section{References}

1. Jiang SJ, Wang SH, Sun YJ and Zhang Q: Medicinal properties of Hericium erinaceus and its potential to formulate novel mushroom-based pharmaceuticals. Appl Microbiol Biotechnol 98: 7661-7670, 2014.

2. Shimokawa $\mathrm{H}$ and Satoh K: Light and dark of reactive oxygen species for vascular function: 2014 ASVB (Asian Society of Vascular Biology). J Cardiovasc Pharmacol 65: 412-418, 2015.

3. Holmes C: Review: Systemic inflammation and Alzheimer's disease. Neuropathol Appl Neurobiol 39: 51-68, 2013.

4. Kang KA, Kim KC, Bae SC and Hyun JW: Oxidative stress induces proliferation of colorectal cancer cells by inhibiting RUNX3 and activating the Akt signaling pathway. Int J Oncol 43: 1511-1516, 2013.

5. Alzoghaibi MA: Concepts of oxidative stress and antioxidant defense in Crohn's disease. World J Gastroenterol 19: 6540-6547, 2013.

6. Abdullah N, Ismail SM, Aminudin N, Shuib AS and Lau BF: Evaluation of selected culinary-medicinal mushrooms for antioxidant and ACE inhibitory activities. Evid Based Complement Alternat Med 2012: 464238, 2012.

7. Fui HY, Shieh DE and Ho CT: Antioxidant and free radical scavenging activities of edible mushrooms. Journal of Food Lipids 9: 35-46, 2002.

8. Han ZH, Ye JM and Wang GF: Evaluation of in vivo antioxidant activity of Hericium erinaceus polysaccharides. Int J Biol Macromol 52: 66-71, 2013.

9. Xu H, Wu PR, Shen ZY and Chen XD: Chemical analysis of Hericium erinaceum polysaccharides and effect of the polysaccharides on derma antioxidant enzymes, MMP-1 and TIMP-1 activities. Int J Biol Macromol 47: 33-36, 2010.

10. Khan MA, Tania M,Liu R and Rahman MM: Hericium erinaceus: An edible mushroom with medicinal values. J Complement Integr Med 10: 253-258, 2013.

11. Snitsarev V, Young MN, Miller RM and Rotella DP: The spectral properties of (-)-epigallocatechin 3-O-gallate (EGCG) fluorescence in different solvents: Dependence on solvent polarity. PLoS One 8: e79834, 2013.
12. Jiang S, Ma Y and Yan D: Antioxidant and antimicrobial properties of water soluble polysaccharide from Arachis hypogaea seeds. J Food Sci Technol 51: 2839-2844, 2014.

13. Negro C, Tommasi L and Miceli A: Phenolic compounds and antioxidant activity from red grape marc extracts. Bioresour Technol 87: 41-44, 2003.

14. Chen $\mathrm{H}$, Yan $\mathrm{M}$, Zhu $\mathrm{J}$ and $\mathrm{Xu} \mathrm{X}$ : Enhancement of exo-polysaccharide production and antioxidant activity in submerged cultures of Inonotus obliquus by lignocellulose decomposition. J Ind Microbiol Biotechnol 38: 291-298, 2011.

15. Shi R, Zhang Q, Vriesekoop F, Yuan Q and Liang H: Preparation of organogel with tea polyphenols complex for enhancing the antioxidation properties of edible oil. J Agric Food Chem 62: 8379-8384, 2014.

16. Vamanu E: In vitro antimicrobial and antioxidant activities of ethanolic extract of lyophilized mycelium of Pleurotus ostreatus PQMZ91109. Molecules 17: 3653-3671, 2012.

17. Moein MR, Moein S and Ahmadizadeh S: Radical scavenging and reducing power of Salvia mirzayanii subfractions. Molecules 13: 2804-2813, 2008.

18. Nahar L, Nasrin F, Zahan R, Haque A, Haque E and Mosaddik A: Comparative study of antidiabetic activity of Cajanus cajan and Tamarindus indica in alloxan-induced diabetic mice with a reference to in vitro antioxidant activity. Pharmacognosy Res 6: 180-187, 2014.

19. Mensor LL, Menezes FS, Leităo GG, Reis AS, dos Santos TC, Coube CS and Leitão SG: Screening of Brazilian plant extracts for antioxidant activity by the use of DPPH free radical method. Phytother Res 15: 127-130, 2001.

20. Rajendran P, Nandakumar N, Rengarajan T, Palaniswami R, Gnanadhas EN, Lakshminarasaiah U, Gopas J and Nishigaki I: Antioxidants and human diseases. Clin Chim Acta 436: 332-347, 2014.

21. Rudbäck J, Bergström MA, Börje A, Nilsson U and Karlberg AT: $\alpha$-Terpinene, an antioxidant in tea tree oil, autoxidizes rapidly to skin allergens on air exposure. Chem Res Toxicol 25: 713-721, 2012.

22. Fritz KS and Petersen DR: Exploring the biology of lipid peroxidation-derived protein carbonylation. Chem Res Toxicol 24: 1411-1419, 2011.

23. Choi BS, Sapkota K, Choi JH, Shin CH, Kim S and Kim SJ: Herinase: A novel bi-functional fibrinolytic protease from the monkey head mushroom, Hericium erinaceum. Appl Biochem Biotechnol 170: 609-622, 2013.

24. Kim KH, Noh HJ, Choi SU and Lee KR: Isohericenone, a new cytotoxic isoindolinone alkaloid from Hericium erinaceum. J Antibiot (Tokyo) 65: 575-577, 2012.

25. Prior RL, Wu X and Schaich K: Standardized methods for the determination of antioxidant capacity and phenolics in foods and dietary supplements. J Agric Food Chem 53: 4290-4302, 2005.

26. Moon JK and Shibamoto T: Antioxidant assays for plant and food components. J Agric Food Chem 57: 1655-1666, 2009.

27. Bhattacharyya A, Chattopadhyay R, Mitra S and Crowe SE: Oxidative stress: An essential factor in the pathogenesis of gastrointestinal mucosal diseases. Physiol Rev 94: 329-354, 2014.

28. Zhou D, Ruan J, Cai Y, Xiong Z,Fu W and Wei A: Antioxidant and hepatoprotective activity of ethanol extract of Arachniodes exilis (Hance) Ching. J Ethnopharmacol 129: 232-237, 2010.

29. Bi J, Huang Y, Xiao Y, Cheng J, Li F, Wang T, Chen J, Wu L, Liu Y, Luo R and Zhao X: Association of lifestyle factors and suboptimal health status: A cross-sectional study of Chinese students. BMJ Open 4: e005156, 2014.

30. Tokarz P, Kaarniranta K and Blasiak J: Role of antioxidant enzymes and small molecular weight antioxidants in the pathogenesis of age-related macular degeneration (AMD). Biogerontology 14: 461-482, 2013.

31. Lim JL, Wilhelmus MM, de Vries HE, Drukarch B, Hoozemans JJ and van Horssen J: Antioxidative defense mechanisms controlled by NRF2: State-of-the-art and clinical perspectives in neurodegenerative diseases. Arch Toxicol 88: 1773-1786, 2014 